\title{
Long-lived magnetoexcitons in 2D-fermion system
}

\author{
L.V. Kulik, A.S. Zhuravlev, A.V. Gorbunov, V.B. Timofeev, and I. V. Kukushkin \\ Institute of Solid State Physics, RAS, Chernogolovka 142432, Russian Federation \\ E-mail: gorbunov@issp.ac.ru
}

Received July 29, 2016, published online November 25, 2016

\begin{abstract}
The paper addresses the experimental technique that, when applied to a 2D-electron system in the integer quantum Hall regime with filling factor $v=2$ (the Hall insulating state), allows resonant excitation of magnetoexcitons, their detection, control of an ensemble of long-lived triplet excitons and investigation of their radiationless decay related to exciton spin relaxation into the ground state. The technique proposed enables independent control of photoexcited electrons and Fermi-holes using photoinduced resonance reflection spectra as well as estimate with a reasonable degree of accuracy the resulting density of photoinduced electron-hole pairs bound into magnetoexcitons. The mere existence of triplet excitons was directly established by inelastic light scattering spectra which were analyzed to determine the value of singlet-triplet exciton splitting. It was found that the lifetimes of triplet excitons conditioned by electron spin relaxation in highly perfect GaAs/AlGaAs heterostructures with highly mobile $2 \mathrm{D}$ electrons are extremely long exceeding $100 \mu$ s at $T<1 \mathrm{~K}$. The paper presents a qualitative explanation of the long-spin relaxation lifetimes which are unprecedented for translationinvariant $2 \mathrm{D}$ systems. This enabled us to create sufficiently high concentrations of triplet magnetoexcitons, electrically neutral excitations following Bose-Einstein statistics, in a Fermi electron system and investigate their collective properties. At sufficiently high densities of triplet magnetoexcitons and low temperatures, $T<1 \mathrm{~K}$, the degenerate magnetofermionic system exhibits condensation of the triplet magnetoexcitons into a qualitatively new collective state with unusual properties which occurs in the space of generalized moments (magnetic translation vectors). The occurrence of a condensed phase is accompanied with a significant decrease in the viscosity of the photoexcited system, which is responsible for electron spin transport at macroscopic distances, as well as with the effects of threshold enhancement of the system response to the external action of the electromagnetic field and emergence of a new intensive radiative recombination channel.
\end{abstract}

PACS: 71.35.Lk Collective effects (Bose effects, phase space filling, and excitonic phase transitions);

73.21.Fg Electron states and collective excitations in multilayers, quantum wells, mesoscopic, and nanoscale systems: Quantum wells;

78.67.De Optical properties of low-dimensional, mesoscopic, and nanoscale materials and structures: Quantum wells.

Keywords: two-dimensional cyclotron singlet and triplet magnetoexcitons, Hall insulator, 2D-fermion system, magnetofermionic condensate.

\section{Introduction}

The interest to the problem of magnetoexciton excitations in fermion systems dates back to the middle of the 60 s of the last century. It is well known that the Coulomb interaction in metals, except its short-range part, is strongly screened. In some cases this residual short-range action turns out to be substantial and should be taken into account in discussion of exciton effects. An example is the socalled edge singularities experimentally discovered in discrete $\mathrm{x}$-ray absorption and emission spectra of metals. This phenomenon results from short-range Coulomb correla- tions and is revealed in the asymmetric form of $K$ - and $L$ lines of $x$-ray alkali metal spectra. These singularities were exactly calculated in the works by Nozieres and De Dominicis [1]. In this connection it is pertinent to note the Mahan singularity that occurs on the edge of the degenerate Fermi spectrum due to Coulomb electron-hole correlations during interband transitions in direct gap semiconductors (see [2,3]). It should be noted, however, that in the case of exciton creation in $3 \mathrm{D}$ metals such states are always unstable and short-lived, which is illustrated by the recent experiments using a femtosecond laser for excitation of extremely short-lived excitons on silver surface [4]. 
However, the situation with lifetimes of specific magnetoexciton excitations is radically different in a 2D metal placed in a sufficiently strong transverse magnetic field. The principle possibility of excitation of the magnetoexciton states in the integer and fractional quantum Hall regimes was previously discussed in literature (see, for instance, [5-7]) along with the possibility of existence of long-lived magnetoexciton excitations [8-10]. Note also theoretical work [11] in which it was shown that in a fairly strong magnetic field the $2 \mathrm{D}$ system should behave almost as a perfect Bose gas.

The aim of the present work is to demonstrate the technique of excitation of long-lived triplet magnetoexcitons in a 2D Fermi system placed in a sufficiently strong transverse magnetic field, to show methods of magnetoexciton detection and control of a magnetoexciton ensemble as well as to demonstrate unexpected novel collective properties of the Fermi system arising under the conditions of excitation of high densities of such magnetoexcitons following Bose-Einstein statistics at sufficiently low temperatures.

Spin-triplet magnetoexcitons are composite bosons, so their ensemble is expected to reveal the Bose-Einstein condensation (BEC) phenomenon. It is known, however, that in two- as well as one-dimensional systems at any arbitrary low but finite temperature the long-range order is destroyed by thermal fluctuations [12]. Therefore, Bose condensate can exist only at $T=0$, which is of no more than theoretical interest. Indeed, in the 2D-case thermal fluctuations do not completely destroy the long-range order: spatial correlations may remain. They decay with distance by the power law rather than exponentially. This turns out to be sufficient for superfluid phase transitions at finite temperature (Berezinsky-Kosterlitz-Thouless (BKT) phase transition $[13,14])$. In this case the superfluid phase transition is caused by formation of topological vortexantivortex defects. There is now abundant experimental evidence of BKT phase transition in different quasi-twodimensional systems: layered superconductors, liquid helium films, Josephson junction arrays, etc.

Berezinsky-Kosterlitz-Thouless phase transition, also known in literature as topological phase transition, connects critical transition temperature $T_{c}$ with condensate density $n_{0}$, particle effective mass $m^{*}$ and is described by the following expression:

$$
k_{B} T_{c}=\frac{\pi}{2} \frac{\hbar^{2} n_{0}}{m_{\mathrm{ex}}^{*}},
$$

where $k_{B}$ is the Boltzmann constant. In our case for triplet magnetoexcitons the values of translation effective mass and experimentally achievable exciton density are: $m_{\mathrm{ex}}^{*} \cong 0.25 m_{0}$ ( $m_{0}$ is the free electron mass) and $n_{0} \leq 2 \cdot 10^{10} \mathrm{~cm}^{-2}$. As a result, using the above formula we obtain the following estimate for critical BKT temperature: $T_{c} \leq 1 \mathrm{~K}$. This means that for the case in question the collective behavior of the triplet cyclotron excitons of the above density should be expected at temperatures near $1 \mathrm{~K}$, which is in accordance with BKT phase transition.

\section{Investigated structures and experimental technique}

High quality GaAs/AlGaAs heterostructures were studied with dark two-dimensional electron mobility in the range of $(5-20) \cdot 10^{6} \mathrm{~cm}^{2} /(\mathrm{V} \cdot \mathrm{s})$. The high quality of the heterostructures is crucial for probing, manipulation and investigation of the properties of long-lived triplet magnetoexcitons. The experiments were performed using heterostructures with single GaAs quantum wells of width's 17 and $35 \mathrm{~nm}$. The electron concentration in the $2 \mathrm{D}$ channel of symmetrically doped quantum wells in the investigated heterostructures was in the range of $5 \cdot 10^{10}-2.5 \cdot 10^{11} \mathrm{~cm}^{-2}$. When working with a single sample characterized by its intrinsic electron concentration, it was possible to measure only one point on the experimental graph of spin-relaxation time as a function of magnetic field.

The structures in question were placed in a liquid ${ }^{3} \mathrm{He}$ cryostat provided with helium vapor pumping. The cryostat, in turn, was placed in a liquid ${ }^{4} \mathrm{He}$ cryostat with a superconducting solenoid. The operated cryosystem enabled to make optical and transport measurements in magnetic fields up to $14 \mathrm{~T}$ at temperatures down to $0.45 \mathrm{~K}$.

The scheme of experiment is shown in Fig. 1(a). Photoexcitation of spectra and spectral measurements were performed using multimode optical fibers with a $400 \mu \mathrm{m}$ quartz core and a numerical aperture of 0.39 . One of the fibers was used to supply laser excitation to the sample, and the other to collect the emission signal from the sample and transfer it onto the entrance slit of a grating spectrometer with a cooled CCD camera. Two frequency-tuned lasers were used: one with a laser linewidth of $20 \mathrm{MHz}$ for resonant photoexcitation of the $2 \mathrm{D}$-electron system, the other with a linewidth of $5 \mathrm{MHz}$ for spectral measurement of photoinduced resonance reflection (PRR), photoluminescence (PL) and resonant inelastic light scattering (ILS). The action of the direct and scattered laser light from the sample and optical fibers surfaces was eliminated with the use of crossed linear polarizers.

The decay kinetics of pulsed PRR was measured with the use of a disc-shaped rotating mechanical chopper with a radial slit (see Fig. 1(a)). With $11 \mathrm{~ms}$ rotation period of the disc the use of the sharp focusing of the laser spot ensured sharpness of the trailing edge of the laser pulse passing through the slit, about $2 \mu \mathrm{s}$. The weak probe laser emission reflected from the sample surface passed through a bandpass interference filter with a bandwidth of $1.1 \mathrm{~nm}$ to suppress the pumping laser light and then was focused on the entrance of an avalanche photodiode operating in the photon counting mode. We used a gatable photon counting system which allowed reproducing the accumu- 

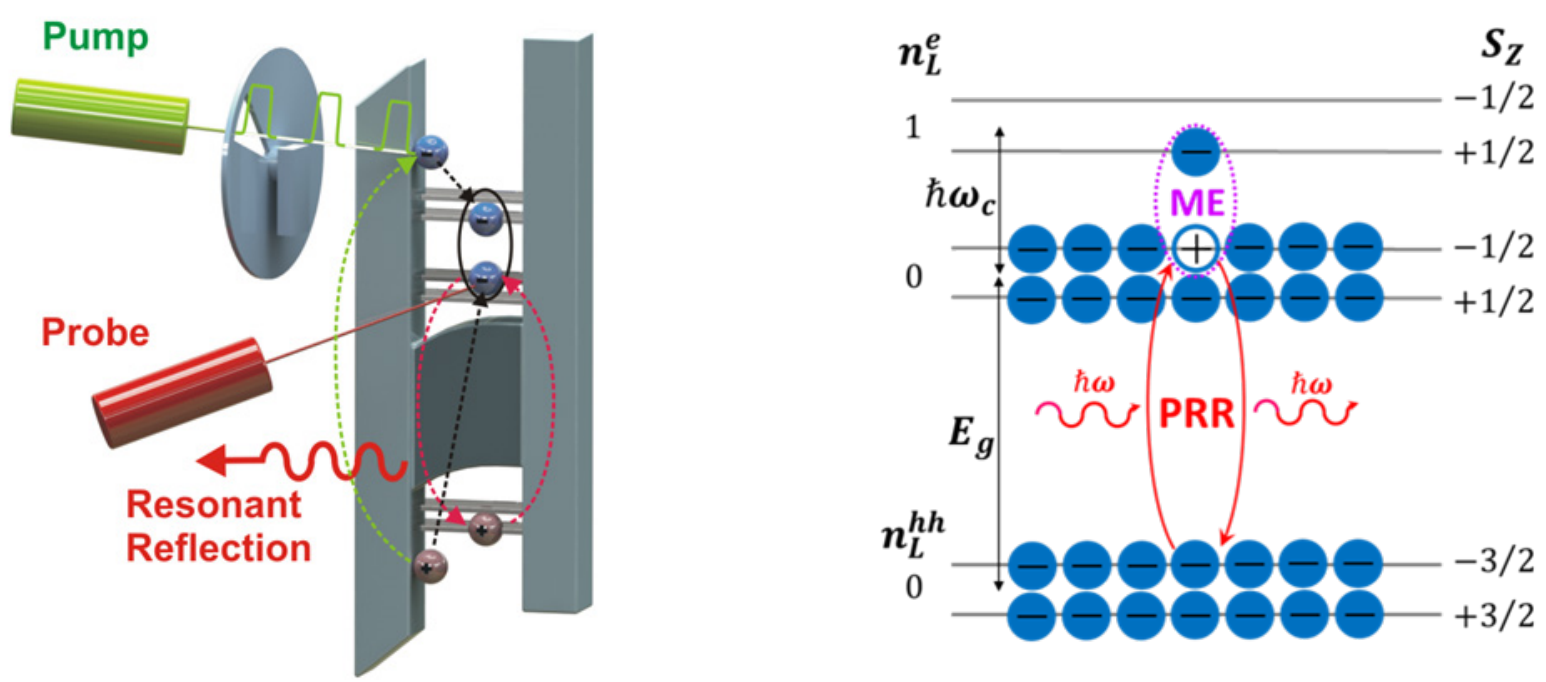

Fig. 1. (Color online) Schemes of experiments (a) and optical transitions (b).

lated PRR signal as a function of time delay with respect to the trailing edge of the excitation pulse. The power of the laser pump exciting electrons on the high Landau levels with quantum numbers $n>1$ did not exceed $0.3 \mathrm{~mW}$, which enabled to avoid overheating effects. The power of the probing laser was less by an order of magnitude.

\section{Experimental results and discussion}

\subsection{Two-dimensional triplet magnetoexcitons}

We have recently studied 2D magnetoexcitons under the conditions of photoexcitation of a Hall insulator with filling factor $v=2$ in a wide symmetrically doped quantum well in a GaAs/AlGaAs heterostructure (see [15]). The research was made using band-to-band excitation that led to formation of a hole in the valence band of the system and an electron on one of the high Landau levels in the conduction band, which was followed by recombination of the valence band hole with an electron of the highest filled magnetic level and rapid relaxation of the photoexcitated electron onto the lowest unfilled Landau level. As a result, Fermi-holes (electron vacancies) appeared on the ground electron cyclotron level $\left(n_{L}^{e}=0\right)$ which are bound into excitons with the photoexcited electrons of the nearest electron cyclotron level $\left(n_{L}^{e}=1\right)$. The optical transition scheme is presented in Fig. 1(b). Such excitons will be further called cyclotron magnetoexcitons (ME). Owing to the spin degrees of freedom of electrons and holes, there are two types of cyclotron ME: singlet and triplet ME. Singlet excitons have resulting spin $S=0$, and, in accordance with the Kohn theorem [16] and for symmetry reasons, their excitation energy is exactly equal to cyclotron energy $\hbar \omega_{c}$. The singlet exciton is also called a spinless magnetoplasmon. The radiative lifetime of the singlet exciton is determined by dipole-allowed recombination of an electron of the first cyclotron level $\left(n_{L}^{e}=1\right)$ with a Fermi-hole of the ground electron level $\left(n_{L}^{e}=0\right)$ with cyclotron photon emission. The characteristic times of such radiative recombination are well below a nanosecond [17].

Triplet cyclotron magnetoexcitons have total spin $S=1$, and the spin projections along the magnetic field direction are $S_{Z}=-1,0,+1$. As contrasted to singlet excitons, also called "bright", triplet excitons are "dark" since their radiative annihilation and direct light excitation are strictly forbidden within the dipole approximation. Yet, for triplet excitons there are no limitations owing to symmetry reasons related to the Kohn theorem. Hence, they have binding energy conditioned by the Coulomb electron-hole interaction. Note that singlet-triplet splitting vanishes at $k=0$ only in the first order of the perturbation theory by the small parameter of the Coulomb-to-cyclotron energy ratio. In the second order of the perturbation theory the splitting reaches fractions of $\mathrm{meV}$ in the magnetic fields in question $[8,9]$. It is this splitting that is responsible for the spin-triplet cyclotron ME becoming a lowest-energy excitation in all even integer quantum Hall states [8,9]. Of great interest is the problem of triplet $\mathrm{ME}$ dispersion in the wave pseudovector function which was considered in theoretical works [6,22]. For symmetry reasons the triplet 2D ME should possess the orbital properties of the $p$-electron state. Therefore, the energy minimum of such an electron state on the dispersion curve should be in the range of the values of pseudovector $\tilde{k} \cong l_{H}^{-1}$, where $l_{H}$ is magnetic length, i.e., it should have a roton character. This is qualitatively demonstrated in Fig. 2. As at low temperatures triplet magnetoexcitons accumulate in region $k \neq 0$, their decay cannot proceed radiatively and requires participation of phonons which can take away an exciton momentum of the inverse magnetic length scale. Relaxation of the integer spin triplet exciton occurs as a consequence of spin-orbit coupling that is not significant in GaAs heterostructures. Thus, a priori we can confidently state that the lifetimes of triplet magnetoexcitons and the time-related spin relaxation should be sufficiently longtime processes. 


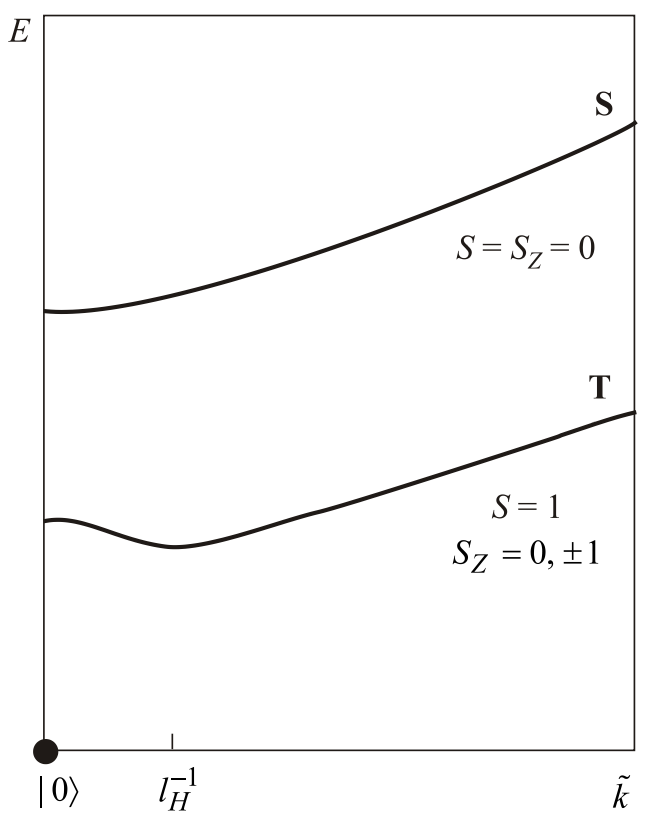

Fig. 2. Dispersion curves of two-dimensional singlet and triplet magnetoexcitons according to paper [22].

We have developed an experimental technique [15] that enables photoexcitation of an ensemble of translationinvariant, i.e., free, triplet cyclotron magnetoexcitons, control of their parameters and investigation of the kinetics of exciton relaxation into the ground state (measurement of radiationless magnetoexciton lifetime). To achieve control of photoexcited electrons and Fermi-holes which finally couple to form triplet magnetoexcitons, studies were made of resonant photoreflection (RR) using dipole-allowed optical transitions between the discrete Landau levels of the valence band and conduction band with $2 \mathrm{D}$ electrons in a quantum well. An a priori assumption can be made that when testing a Hall insulator with filling factor $v=2$ there should be no resonant reflection signal corresponding to transition from the zeroth Landau level of valence heavy hole $\left(n_{L}^{h}=0\right)$ to the zeroth electron Landau level $\left(n_{L}^{e}=0\right)$ since all the electron states of the ground electron cyclotron level are completely filled. Yet, on switching of the optical pump exciting electrons towards the upper Landau levels $\left(n_{L}^{e}>1\right.$, see the transition scheme in Fig. 1(b)) at sufficiently low temperature, it would be reasonable to expect formation of triplet excitons with spin projection $S_{Z}=-1$, since it is these excitons which make up the lowest excited state in the 2D-electron system in the magnetic field. Therefore, the process of triplet exciton formation should manifest itself in reduction of unfilled states (Fermi-holes) of the first electron Landau level and simultaneous occurrence of unfilled states (Fermi-holes) on the zeroth electron level. It has been already agreed that such expected changes in reflection spectra are called PRR. Two peaks should be observed in PRR spectra: a positive peak corresponding to optical transition from the zeroth cyclotron level of heavy holes to the upper spin sublevel of the zeroth cyclotron level of 2D electrons, (0-0) transition, and a negative peak corresponding to transition from the first cyclotron level of valence-band heavy holes to the first electron cyclotron level, (1-1) transition. Thus, the proposed PRR method is an indirect way of testing triplet (optically inactive!) excitons using optically allowed transitions between discrete Landau levels of valence and conduction bands, i.e., (0-0) and (1-1) transitions.

Now let us turn to the experimental results. Figure 3 presents PL and PRR spectra measured experimentally at filling factor $v=2$ in photoexcitation conditions. First let us consider the luminescence spectrum (blue curve) that exhibits a pronounced circularly polarized doublet $\left(\sigma^{+}-\sigma\right)$ corresponding to the electronic $(0-0)$ transitions between the spin components of the heavy holes in the valence band and those of the electrons in the conduction band. The value of doublet splitting closely corresponds to the sum of spin splitting of the ground cyclotron level of valence heavy holes and that of the ground cyclotron level of 2D electrons. In Fig. 3 the PRR is represented by the red curve. The PRR spectrum shows a positive peak in the electronic (0-0)-transition region and a negative peak in the electronic (1-1)-transition region, which is in full agreement with the expectations expressed above. Such behavior of the PRR spectra is due to formation of lowenergy triplet magnetoexcitons containing electrons of the first electron Landau level bound by Coulomb interaction to the Fermi-holes of the zeroth electron level (see the transition scheme in Fig. 1(b)). Thus, studies of the influence of nonequilibrium Fermi-holes on the RR signal enable to infer the behavior of the whole magnetoexciton en-

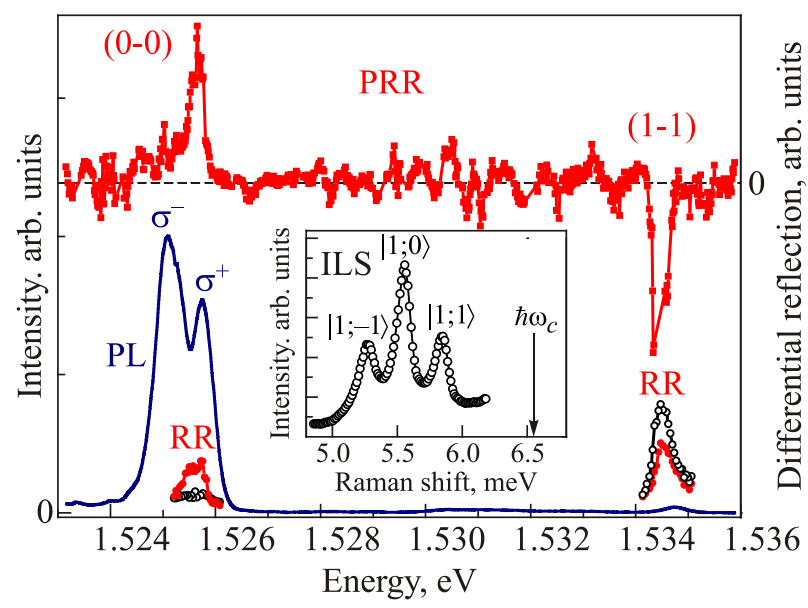

Fig. 3. (Color online) Photoluminescence (PL) and photoinduced resonant reflection (PRR) spectra measured at filling factor $v=2$ in a $17 \mathrm{~nm}$ quantum well (mobility $5 \cdot 10^{6} \mathrm{~cm}^{2} /(\mathrm{V} \cdot \mathrm{s}$ ), electron concentration $2.4 \cdot 10^{11} \mathrm{~cm}^{-2}$ ) in $5 \mathrm{~T}$ perpendicular magnetic field at $0.45 \mathrm{~K}$. The inset shows an inelastic light scattering (ILS) spectrum corresponding to scattering on the cyclotron triplet magnetoexciton mode measured in similar experimental conditions. Below are shown the resonant reflection (RR) spectra with (solid symbols) and without (empty symbols) extra photoexcitation. 
semble. It should be emphasized that photoexcitation of magnetoexcitons takes place in a degenerate Fermi electron system.

Although triplet excitons are not optically active, their existence can be directly established by ILS spectra (see the inset in Fig. 3) and use them for indirect determination of the value of singlet-triplet exciton splitting [9]. Singlettriplet exciton splitting is measured as the energy difference between a singlet exciton and the exciton triplet center of weight (the spectral position of spin component $S_{Z}=0$ ) observed in ILS spectra experiments. This splitting appeared to be comparatively large, about $1 \mathrm{meV}$ [9], which may seem surprising because in bulk GaAs crystals singlet-triplet exciton splitting is very small and comes to only $20 \mu \mathrm{eV}[18,19]$. However, first, it should be remembered that the matrix elements of the exchange interband Coulomb interaction are suppressed compared to intraband exchange owing to the difference in the Bloch functions of electrons in different bands. Second, given quantum confinement (spatial restrictions), singlet-triplet exciton splitting increases significantly due to the increasing exchange electron-hole interaction. For instance, in fairly narrow GaAs quantum wells such splitting reaches approximately $150 \mu \mathrm{eV}$ [18]. In the considered case of 2D cyclotron magnetoexcitons in the Hall insulator the increase in the singlet-triplet exciton splitting is still more significant reaching $1 \mathrm{meV}$ due to the transverse magnetic field (up to $10 \mathrm{~T}$ ) that ensures a strong spatial confinement for the carriers.

We have studied the behavior of radiationless lifetime of triplet magnetoexcitons which is directly related to exciton spin relaxation as functions of temperature and magnetic field in quantum wells of different widths. To this end, research was made of the kinetics of PRR under pulsed photoexciation conditions using a mechanical chopper. The decay kinetics of the PRR measured for two quantum wells of different widths in the optical (0-0)-transition region is presented in Fig. 4. It is seen that the kinetics of the PRR signals is exponential and the relaxation times found from the kinetics are super long reaching tens of microseconds. We should emphasize that the kinetics of the PRR signal rise in the (1-1)-transition region occurs over the exactly same time as for the (0-0) transition, which points to common relaxation dynamics for the exciton states formed by electrons on the first and by Fermiholes on the zeroth Landau levels. This observation is the additional confirmation concerning cyclotron magnetoexciton creation by photoexcitation.

Very informative is the temperature dependence of the relaxation rate shown in Fig. 5(a). It is seen that the temperature dependence of the relaxation rate is exponential at temperatures $T>1 \mathrm{~K}$ with characteristic relaxation time $\tau_{1} \cong 1 \mathrm{~ns}$ and activation energy gap $\Delta \cong 11 \mathrm{~K}$. Such behavior indicates that this temperature range involves an activation relaxation channel including electron spin-flip processes caused by spin-orbit coupling, an increase in exciton ener-

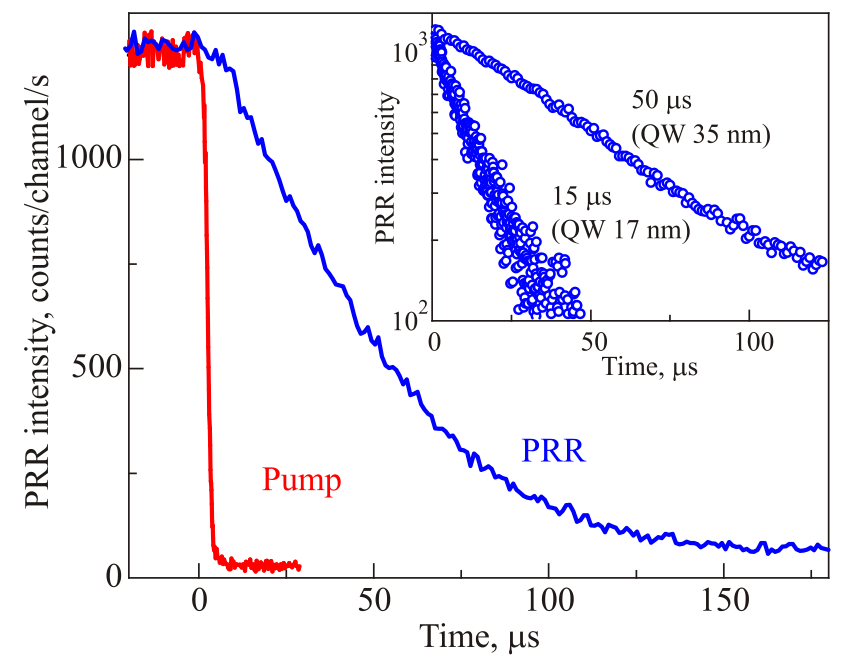

Fig. 4. (Color online) Typical time dependence of photoinduced resonant reflection (PRR) decay. The instrument function is marked in red. The inset presents two kinetic dependences measured for $35 \mathrm{~nm}$ (long decay time) and $17 \mathrm{~nm}$ (short decay time) wide quantum wells with filling factor $v=2$ in the $4 \mathrm{~T}$ magnetic field at $0.45 \mathrm{~K}$. The electron concentration in both quantum wells was $2.0 \cdot 10^{11} \mathrm{~cm}^{-2}$, the mobilities being $1.5 \cdot 10^{7} \mathrm{~cm}^{2} /(\mathrm{V} \cdot \mathrm{s})$ and $5 \cdot 10^{6} \mathrm{~cm}^{2} /(\mathrm{V} \cdot \mathrm{s})$, respectively.

gy by the value of cyclotron energy and subsequent emission of a photon with cyclotron energy [10]. Under these conditions the measured activation gap $\Delta$ is nothing but the Coulomb triplet exciton binding energy that is exactly equal to the energy of singlet-triplet exciton splitting. This Coulomb energy independently measured by the ILS spectra approximately coincides with the found activation gap $\Delta$. In the low-temperature region, $T<1 \mathrm{~K}$, the PRR kinetics is no longer temperature dependent (Fig. 5(a)). Hence, it is a temperature range of a change of the relaxation mechanism that is obviously no longer thermally activated. We believe that in the low-temperature range a radiationless decay of triplet cyclotron magnetoexcitons takes place which is accompanied with relaxation of the resulting spin and generation of the short-wave acoustic phonons to which the triplet exciton energy is transferred. Such a relaxation mechanism was earlier proposed and analyzed by S. Dickmann in the paper [10]. In particular, the theory [10] predicts that in this case relaxation time $\tau_{0}$ should be superlinearly dependent on the extent of the electron wave function in the Z-direction of heterostructure growth. To verify the prediction we investigated two quantum wells 17 and $35 \mathrm{~nm}$ wide with approximately equal concentrations of $2 \mathrm{D}$ electrons. The halfwidths of the envelope electron wave functions in these quantum wells differed approximately twofold. Figure 5(b) presents the result of relaxation time measurements in these quantum wells which is in good qualitative agreement with the theoretical predictions. Figure 5(b) also confirms another theoretical conclusion: the relaxation rate decreases with increasing magnetic field 

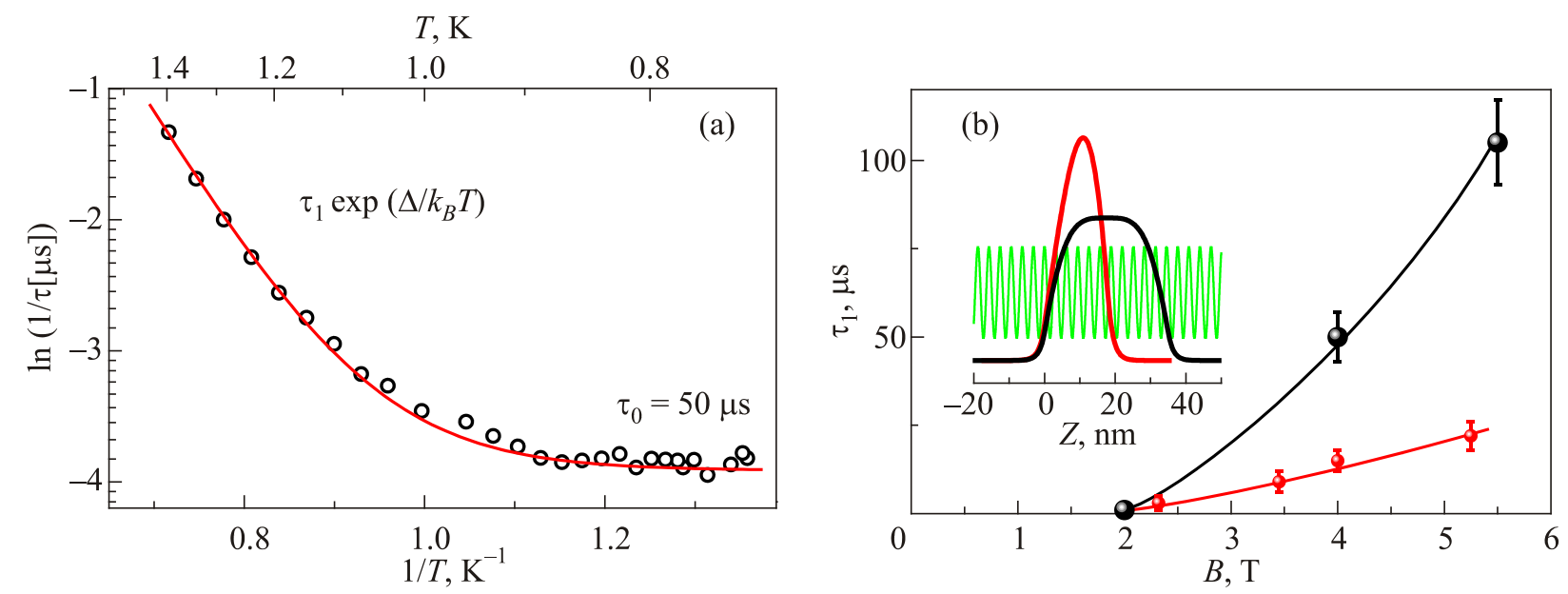

Fig. 5. (Color online) (a) Temperature dependence of relaxation time measured in the $35 \mathrm{~nm}$ wide quantum well at filling factor 2 in the $4 \mathrm{~T}$ magnetic field and fitting curve with an activation behavior and saturation in the low-temperature limit. (b) Summary plot of lowtemperature relaxation time as a function of magnetic field. The small symbols correspond to the data measured in the $17 \mathrm{~nm}$ wide quantum well and the large symbols to those measured in the $35 \mathrm{~nm}$ quantum well. The inset shows comparison of the electron wave functions in the quantum wells of different width with the phonon wave function which illustrates the significant enhancement of the phonon emission process in the narrow wells.

since the electron-phonon interaction diminishes substantially for harder acoustic phonons with frequencies close to cyclotron frequency $\omega_{c}$. There are good reasons to believe that generally the experiment exhibits good agreement with the theoretical models though the theory quantitatively predicts much longer relaxation times than those experimentally observed. The possible reason for the discrepancies is likely related with the inexact knowledge of the envelope electron wave functions in the Z-direction of quantum well growth to which the matrix element of the electron-phonon interaction is extremely sensitive.

Thus, the direct measurements of the kinetics of PRR at weak pulsed pumping made it possible to establish that at low temperatures $T<<T_{S T}\left(k_{B} T_{S T}\right.$ is the energy of singlettriplet splitting) the lifetimes of triplet excitons which are closely related to the spin relaxation of the whole electron system are extremely large reaching hundreds of microseconds.

\subsection{Magnetofermion condensate}

Long lifetimes (about 100 microseconds) enabled to create densities of photoexcited long-lived triplet magnetoexcitons exceeding $10^{10} \mathrm{~cm}^{-2}$ (the maximum fraction of the photocreated excitons was about $10 \%$ of the number of states on one spin Landau sublevel). With such lifetimes one may expect that the collective phenomena related to condensation of triplet magnetoexcitons in a magnetic fermion system will proceed in quasiequilibrium conditions. In our preliminary experiments it was found that, when the temperature was lowered below $1 \mathrm{~K}$, the PRR signal related with a sufficiently dense system of triplet magnetoexcitons increases threshold-wise by an order of magnitude, and with further temperature lowering the threshold of the in- crease is shifted towards the region of lower-power optical excitations.

The gigantic increase of the optical response is associated with formation of condensate clusters of long-lived triplet magnetoexcitons interacting coherently with the external electromagnetic field (superabsorption). The radiative recombination channel of the electrons of the Fermi level is enhanced simultaneously with the increasing optical response of the condensed magnetoexciton ensemble. This suggests occurrence of a collective phase in which not only magnetoexcitons but also the electrons under the Fermi level within the coherence length scale are ordered into clusters which, when interacting with the electromagnetic field, behave collectively and are responsible for intense luminescence (superemission) in close analogy with the Dicke effect [20]. Under high enough photoexcitations and rather low temperature, $T \cong 0.5 \mathrm{~K}$, we have observed in the spectral range corresponding to $(0-0)$ transitions a new very intense photoluminescence line which strongly dominates over other radiative channels in this spectral region and appears abruptly under variation of temperature or excitation power. We have associated the appearance of this new very intense PL channel with creation of condensed phase in magnetofermion system with magnetoexcitons. Hence, the dense magnetoexciton ensemble induces transformation of the nonequilibrium 2D-electron system into a phase of "superabsorbing" and "superemitting" multiparticle clusters. Most of the effects observed are qualitatively explained from the viewpoint of formation of a spatially limited coherent state of spin triplet magnetoexcitons, and the newly formed phase itself is the first example of a 2D-fermionic system with essentially collective Bose properties. 
The measurement of the temperature of the thresholdlike increase of photoinduced triplet magnetoexcitons as a function of optical pump made it possible to plot a phase diagram separating the region of triplet magnetoexciton gas and that of strongly correlated magnetic fermion condensate, what is in the focus of our nearest interests.

\subsection{Effect of "superfluidity" of magnetofermion condensate}

A detailed investigation revealed an unexpectedly nonmonotonic behavior of relaxation time of triplet magnetoexcitons occurring with a decrease in temperature (fixed pump) or an increase in pump power (fixed temperature). First the relaxation time of magnetoexcitons increases threshold-wise by more than an order of magnitude, which is due to formation of a condensed phase, but then it starts decreasing significantly. Such nonmonotonic behavior points to a threshold-like change in the viscosity of the condensed subsystem of nonequilibrium triplet magnetoexcitons on their diffusion towards the relaxation centers. This naturally suggested a hypothesis of "superfluid flow" of triplet magnetoexciton condensate which was experimentally verified with the use of spatial separation of the regions of photoexcitation and RR detection. The experiments employed a technique with several spatially separated optical fibers. One of the fibers was used to direct emission exciting a magnetoexciton ensemble in a $\sim 100 \mu \mathrm{m}$ sized spot on the sample surface. Two other fibers were placed at $a \approx 2 \mathrm{~mm}$ distance from the first one and used to measure magnetoexciton Fermi-holes in the discrete electron spectrum by means of PRR. The PRR signal in the spatially separated optical fibers was produced only in the conditions of triplet magnetoexciton condensation (low temperatures and rather high pump power) in the region of excitation created by the first fiber. The occurrence of a PRR signal far away from the first fiber means that the excited magnetoexcitons spread out over a distance of $2 \mathrm{~mm}$, which cannot be explained within the common classical diffusion conceptions. The threshold changes in the diffusion properties agree with the concepts of magnetoexciton condensation in pseudo-momentum space. Indeed, triplet magnetoexcitons are $p$-type excitons, and their dispersion contains a roton minimum [6]. When magnetoexcitons are in an incoherent state, the absolute values of their dipole momenta are approximately equal (close to the value corresponding to the roton minimum), yet the spatial orientation of the dipoles is quite random. At sufficiently low temperatures and low densities each magnetoexciton finds its localization site in the fluctuating large-scale chaotic potential that is always present even in highly perfect heterostructures. Triplet magnetoexcitons remain in such localization sites till full relaxation into the ground state. When it is considered that all the pseudo-momenta are proportional to the dipole momenta of magnetoexcitons, their condensation in the pseudo-momentum space should be attended with occurrence of a dipole momentum, the result of summation of the unidirectional momenta of all the magnetoexcitons in the condensate.

\section{Conclusion}

In conclusion it should be noted that $2 \mathrm{D}$ magnetofermion condensate in GaAs/AlGaAs semiconductor heterostructures and the phenomenon of superconductivity in metals exhibit certain general properties despite apparent essential differences. The principal general feature is related in both cases to the presence of a "diluted" boson component in the degenerate Fermi system. In the case in question the role of such a subsystem is played by magnetoexcitons, composite bosons, which are stable quasiparticles owing to Coulomb electron-hole attraction. In a conventional superconductor the boson subsystem is connected with Cooper pairs in which electrons are bound by the electron-phonon interaction. Here it is reasonable to draw an analogy with triplet magnetoexcitons and triplet Cooper pairs in superconductors [21]. The difference, however, is that a metal superconductor is a thermodynamically equilibrium system while magnetofermion condensate, being photoexcited, is metastable but, owing to long lifetimes of triplet magnetoexcitons, it can stay long enough time in a quasi-equilibrium state. Lastly, it should be noted that in superconductors electron Cooper pairs perform dissipationless charge transport, i.e., they are dissipationless flow of charge carriers, whilst in the considered case of magnetofermion condensate magnetoexcitons perform dissipationless transport of an integer electron spin over macroscopic distances. Further experimental and theoretical studies are required to finally clarify the issues considered.

This work is intended to mark the jubilee of I.M. Lifshits, an outstanding theoretical physicist, who made an invaluable fundamental contributions to physics of Fermi systems in condensed media.

Authors acknowledge financial support by Russian Science Foundation, Grant No. 16-12-10075.

1. P. Nozieres and C.T. De Dominicis, Phys. Rev. 178, 1097 (1969).

2. G.D. Mahan, Phys. Rev. 163, 612 (1967).

3. G.D. Mahan, Solid State Physics, v. 29, F. Seits, D. Turnbull, and H. Ehrenreich (eds.), New York: Academic (1974), p. 75.

4. X. Cui, C. Wang, A. Argondizzo, S. Garrett-Roe, B. Gumhalter, and H. Petek, Nature Phys. 10, 505 (2014).

5. Yu.A. Bychkov, S.V. Iordanskii, and G.M. Eliashberg, Pis'ma v Zh. Eksp. Teor. Fiz. 33, 152 (1981) [JETP Lett. 33, 143 (1981)].

6. C. Kallin and B.I. Halperin, Phys. Rev. B 30, 5655 (1984).

7. J.K. Jain, Phys. Rev. Lett. 63, 199 (1989); Adv. Phys. 41, 105 (1992).

8. S. Dickmann and I.V. Kukushkin, Phys. Rev. B 71, 241310(R) (2005). 
9. L.V. Kulik, I.V. Kukushkin, S. Dickmann, V.E. Kirpichev, A.B. Van'kov, A.L. Parakhonsky, J.H. Smet, K. von Klitzing, and W. Wegscheider, Phys. Rev. B 72, 073304 (2005).

10. S.M. Dickmann, Phys. Rev. Lett. 110, 166801 (2013).

11. I.V. Lerner and Yu.E. Lozovik, Zh. Eksp. Teor. Fiz. 80, 1488 (1981) [Sov. Phys. JETP 53, 763 (1981)].

12. P.C. Hohenberg, Phys. Rev. 158, 383 (1967); N. Mermin and H. Wagner, Phys. Rev. Lett. 17, 1133 (1966).

13. V.L. Berezinsky, Zh. Eksp. Teor. Fiz. 59, 907 (1970) [Sov. Phys. JETP 32, 493 (1970)]; Zh. Eksp. Teor. Fiz. 61, 1144 (1971) [Sov. Phys. JETP 34, 610 (1971)].

14. J.M. Kosterlitz and D.J. Thouless, J. Phys. C 6, 1181 (1973).

15. L.V. Kulik, A.V.Gorbunov, A.S. Zhuravlev, V.B. Timofeev, S.M. Dickmann, and I.V. Kukushkin, Nature Sci. Rep. 5, 10354 (2015).

16. W. Kohn, Phys. Rev. 123, 1242 (1961).
17. Q. Zhang, T. Arikawa, E. Kato, J.L. Reno, W. Pan, J.D. Watson, M.J. Manfra, M.A. Zudov, M. Tokman, M. Erukhimova, A. Belyanin, and J. Kono, Phys. Rev. Lett. 113, 047601 (2014).

18. E. Blackwood, M.J. Snelling, R.T. Hartley, S.R. Andrews, and C.T.B. Foxon, Phys. Rev. B 50, 14246 (1994).

19. M. Bayer, A. Kuther, A. Forchel, A.V. Gorbunov, V.B. Timofeev, F. Schafer, J.P. Reithmaier, T.L. Reinecke, and S.N. Walck, Phys. Rev. Lett. 82, 1748 (1999).

20. R.H. Dicke, Phys. Rev. 93, 99 (1954).

21. X.L. Wang, A. Di Bernardo, N. Banerjee, A. Wells, F.S. Bergeret, M.G. Blamire, and J.W.A. Robinson, Phys. Rev. B 89, 140508(R) (2014); A. Di Bernardo, S. Diesch, Y. Gu, J. Linder, G. Divitini, C. Ducati, E. Scheer, M.G. Blamire, and J.W.A. Robinson, Nature Commun. 6, 8053 (2015).

22. I.V. Lerner and Yu.E. Lozovik, Zh. Eksp. Teor. Fiz. 78, 1167 (1980) [Sov. Phys. JETP 51, 588 (1980)]. 